June 23, 2021

\title{
Fluctuations of the heat flux of a one-dimensional hard particle gas
}

\author{
Éric Brunet, Bernard Derrida and Antoine Gerschenfeld \\ Laboratoire de Physique Statistique, Ecole Normale Supérieure, UPMC Paris 6, \\ Université Paris Diderot, CNRS, 24 rue Lhomond, 75231 Paris Cedex 05 - France
}

\begin{abstract}
Momentum-conserving one-dimensional models are known to exhibit anomalous Fourier's law, with a thermal conductivity varying as a power law of the system size. Here we measure, by numerical simulations, several cumulants of the heat flux of a one-dimensional hard particle gas. We find that the cumulants, like the conductivity, vary as power laws of the system size. Our results also indicate that cumulants higher than the second follow different power laws when one compares the ring geometry at equilibrium and the linear case in contact with two heat baths (at equal or unequal temperatures).

keywords: current fluctuations, anomalous Fourier law, hard particle gas

PACS numbers: 02.50.-r, 05.40.-a, 05.70 Ln, 82.20-w
\end{abstract}

\section{Introduction}

Understanding the fluctuations of the flux of heat or of the current of particles through systems in their steady state is a central question in non equilibrium statistical mechanics. Since the discovery of the fluctuation theorem [1-6], one knows that the probability distribution of these fluctuations has some symmetry properties related to time-reversal symmetry. In most cases, the calculation of this distribution for a given microscopic model remains, however, a challenging issue.

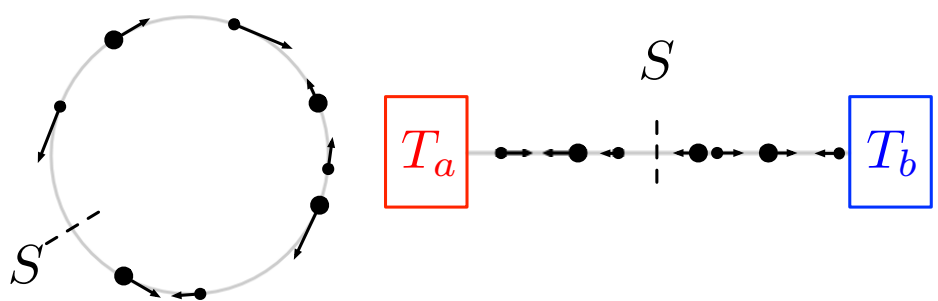

a)

b)

FIG. 1: We measure the cumulants of the integrated current $Q_{t}$ of a one-dimensional hard particle gas with alternating masses 1 and $m_{2}>1$ : fig. 1 a) for an even number of particles on a ring; fig. 1 b) for an odd number of particles in contact with two heat baths (open system). In our simulation, we measure the flux $Q_{t}$ through a section $S$, located anywhere on the ring in fig. 1 a) and half-way between the two heat baths in fig. 1. b); we also measure the flux $\bar{Q}_{t}$ averaged over the whole system.

Over the last few years, several exact expressions for the distribution of these fluctuations have nevertheless been obtained for diffusive systems, such as lattice gases [7-14] (for instance the one-dimensional symmetric simple exclusion process). For diffusive systems in their steady state, in the two geometries of fig. 1 (the ring geometry in equilibrium at temperature $T$ and the open system, i.e. a finite system in contact with two heat baths at temperatures $T_{a}$ and $T_{b}$ ), the cumulants $\left\langle Q_{t}^{n}\right\rangle_{c}$ of the flux of energy $Q_{t}$ during a long time $t$ take, for a large system size $L$, the following form:

$$
\lim _{t \rightarrow \infty} \frac{1}{t}\left\langle Q_{t}^{2}\right\rangle^{\text {ring }} \simeq \frac{1}{L} A(T) ; \quad \lim _{t \rightarrow \infty} \frac{1}{t}\left\langle Q_{t}^{2 n}\right\rangle_{c}^{\text {ring }} \simeq \frac{1}{L^{2}} B_{n}(T) \text { for } n \geq 2 ; \quad \lim _{t \rightarrow \infty} \frac{1}{t}\left\langle Q_{t}^{n}\right\rangle_{c}^{\text {open }} \simeq \frac{1}{L} C_{n}\left(T_{a}, T_{b}\right)
$$

For the ring geometry (fig. 1 a), explicit expressions of the prefactors $A(T)$ and $B_{n}(T)$ have been obtained for the symmetric simple exclusion process, as well as for generic diffusive systems with one conserved quantity [10]. For the open case (fig. 1 b), the amplitudes $C_{n}\left(T_{a}, T_{b}\right)$ are also known for the one-dimensional symmetric exclusion process [7 - 9] (with expressions identical to those which had been previously determined for disordered one-dimensional conductors [15, 16]); they are also known for generic diffusive systems with one conserved quantity [11-14, 17-19].

The $1 / L$ dependence of $\left\langle Q_{t}\right\rangle^{\text {open }} / t$ for the open case means that diffusive systems satisfy Fourier's law [20]. Together with the fluctuation-dissipation theorem, Fourier's law also implies the $1 / L$ decay of $\left\langle Q_{t}^{2}\right\rangle^{\text {ring }} / t$ (or of $\left\langle Q_{t}^{2}\right\rangle^{\text {open }} / t$ for $T_{a}=T_{b}$ ). 
It is remarkable that the $L$ dependence (1) of all the cumulants is generic for diffusive systems, with a few exceptions where some prefactors may vanish and the decay with $L$ is faster [10]. All these $L$ dependencies follow from the fact that, for large diffusive systems, local equilibrium holds and can be treated within the macrosopic fluctuation theory (MFT), a theory of diffusive systems which allows one to calculate explicitly a number of properties of non-equilibrium steady states [21-25]. For systems with more than one conserved quantity, much less has been done so far concerning the cumulants of the current but, as long as the system is diffusive, one expects, from the MFT, the same $L$ dependencies of the cumulants as in (1).

Momentum-conserving systems in one dimension are known to exhibit anomalous Fourier's law [26-32], with an average current varying as a non trivial power law of the system size $L$ :

$$
\lim _{t \rightarrow \infty} \frac{1}{t}\left\langle Q_{t}\right\rangle^{\text {open }} \sim L^{\alpha-1} C_{1}\left(T_{a}, T_{b}\right) .
$$

The exponent $\alpha$ is not easy to determine [33-37]. It seems to vary with the systems studied; even for a given system, numerical simulations or theoretical approaches do not always agree. The current consensus is that several universality classes exist depending on the nature of the non-linearity of the forces between the atoms [38, 39].

As major numerical efforts have been already done to determine the exponent $\alpha$, our goal here is to present numerical simulations, not to determine $\alpha$ more accurately, but rather to look at the $L$ dependence of higher cumulants of $Q_{t}$.

\section{MEASUREMENT OF THE CUMULANTS FOR THE HARD PARTICLE GAS}

The system we have decided to simulate is a one-dimensional gas [33, 35, 40] of point particles with hard core interactions. It was mainly chosen for its simple dynamics: masses follow ballistic motions between successive collisions, which are elastic. If the particles were all of equal masses, the velocities of colliding particles would simply be exchanged, and the transport of energy would be the same as for an ideal gas; this is why, as in previous studies of hard particle gases, we chose here a two-mass system, with alternating particles of masses 1 and $m_{2}$.

For the ring geometry, we consider an even number $N$ of masses on a circle of length $L=N$, initially in microcanonical equilibrium at fixed energy $E=N$ and zero total momentum: the total energy and the momentum remain of course conserved by the dynamics.

For the open case in contact with two heat baths at unequal (or equal) temperatures, we take an odd number $N$ of particles in a one-dimensional box of size $L=N$, and we choose the particles closest to the boundaries to be of mass 1 . The heat baths at these boundaries are implemented in the following way: whenever a particle hits a boundary, it is reflected as if a thermalized particle was entering the system from the bath, so that the total number of particles in the system remains $N$.

As the steady state is in general not known in the open case, we started our first sample with an initial condition chosen at random; then, the initial configuration of each new sample was taken to be the final configuration of the previous sample. Therefore, apart from transient effects affecting the first samples (which represent a very small fraction of the total), the initial configurations of our samples are typical of steady state configurations.
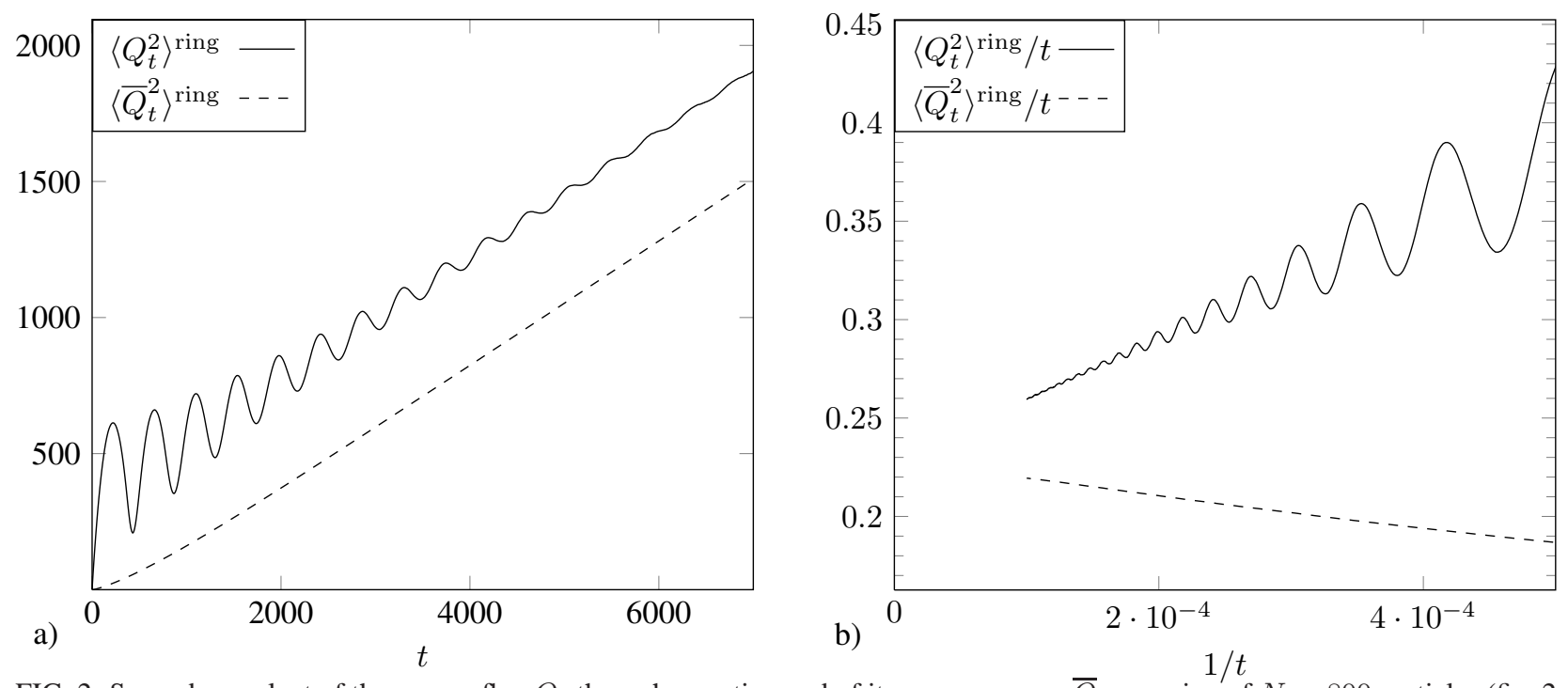

FIG. 2: Second cumulant of the energy flux $Q_{t}$ through a section and of its space average $\bar{Q}_{t}$ on a ring of $N=800$ particles (fig. 2 a). When plotted as functions of $1 / t$ (fig. 2] b), the two ratios $\left\langle Q_{t}^{2}\right\rangle / t$ converge, when $t \rightarrow \infty$, to a common value $\simeq 0.23$. 
Through any section of our system, the flux of energy $Q_{t}$ is the algebraic sum of the kinetic energies of the particles crossing the section $S$ during a time interval $t$. In the steady state, the statistical properties of $Q_{t}$ depend on where the section is located (at least in the open geometry). On the other hand, one expects (under the assumption that the internal energy cannot grow indefinitely) that the long time limit of the ratios $\left\langle Q_{t}^{n}\right\rangle_{c} / t$ are independent of where $Q_{t}$ is measured. In our simulations, we measure for the same samples the flux of energy $Q_{t}$ through a fixed section $S$ located at position $L / 2$ in the open geometry (and anywhere on the ring geometry) and its integrated value $\bar{Q}_{t}$ averaged over the whole system.

As shown in fig. 2 a), the cumulants obtained from these two measurements behave differently at finite time, but both exhibit a linear growth for large $t$. Fig. 2 $2 \mathrm{~b}$ ) shows that, when the ratios $\left\langle Q_{t}^{2}\right\rangle_{c} / t$ are plotted versus $1 / t$, the two sets of data converge to a common value in the long time limit. This was the case for all the cumulants we were able to measure: for all the results shown below, the procedure of fig. 2 $\mathrm{b}$ b) was used to estimate the asymptotic values of the cumulants $\left\langle Q_{t}^{n}\right\rangle_{c} / t$.

Note that the $1 / t$ convergence of fig. 2.b) can easily be understood : if the correlation function $\left\langle J\left(t_{1}\right) J\left(t_{2}\right)\right\rangle_{c}=$ $f\left(t_{2}-t_{1}\right)$ of the energy current $J(t)=\partial_{t} Q_{t}$ decays fast enough in the steady state or at equilibrium, then $\left\langle Q_{t}^{2}\right\rangle_{c}=$ $\iint_{0}^{t} \mathrm{~d} t_{1} \mathrm{~d} t_{2}\left\langle J\left(t_{1}\right) J\left(t_{2}\right)\right\rangle_{c}=2 t \int_{0}^{t} f(\tau) \mathrm{d} \tau-2 \int_{0}^{t} \tau f(\tau) \mathrm{d} \tau$ becomes of the form $A t+B$ in the large time limit, so that $\left\langle Q_{t}^{2}\right\rangle_{c} / t \sim A+B / t$.

Remark: all the cumulants we could measure grow linearly with time for large $t$. On the ring geometry, we only observed this growth when performing microcanonical sampling at fixed total energy $E$ and momentum $P$ (we took $E=N$ and $P=0$ ). When $E$ is allowed to fluctuate (canonical ensemble) while keeping $P=0$, the cumulants exhibit a faster growth $\left(\left\langle Q_{t}^{2 n}\right\rangle \sim t^{n}\right.$ ). This is due to the conservation of $E$ by the dynamics on the ring, which introduces non decaying current-current correlations in the long time limit.

\section{SIZE DEPENDENCE OF THE CUMULANTS}

Fig. 3 and 4 show the asymptotic values of $\left\langle Q_{t}^{n}\right\rangle_{c} / t$ we obtained for $1 \leq n \leq 4$. The cumulants were calculated by averaging over a number of samples varying from $2 \cdot 10^{8}$ for $N=50$ to $2 \cdot 10^{6}$ for $N=800$, for the following systems:

- a ring of $50 \leq N \leq 800$ particles with total kinetic energy $N$ and total momentum 0 ;

- an open system of $51 \leq N \leq 801$ particles between two heat baths at temperatures $T_{a}=2$ and $T_{b}=1$;

- the same open system between two heat baths at the same temperature $T_{a}=T_{b}=1$.

In all cases, the particle density $N / L$ was exactly 1 . The mass of the heavier particles, $m_{2}$ was taken to be 2.62 (as in [33]); when we repeated some of our simulations with a different mass ratio, $m_{2}=(1+\sqrt{5}) / 2$ as in [35], we obtained qualitatively similar results (not shown here).

The fourth cumulant of $Q_{t}$ is of course the hardest to obtain: we were only able to measure the asymptotic value of $\left\langle Q_{t}^{4}\right\rangle_{c} / t$ accurately for $N \leq 201$ in the open case.

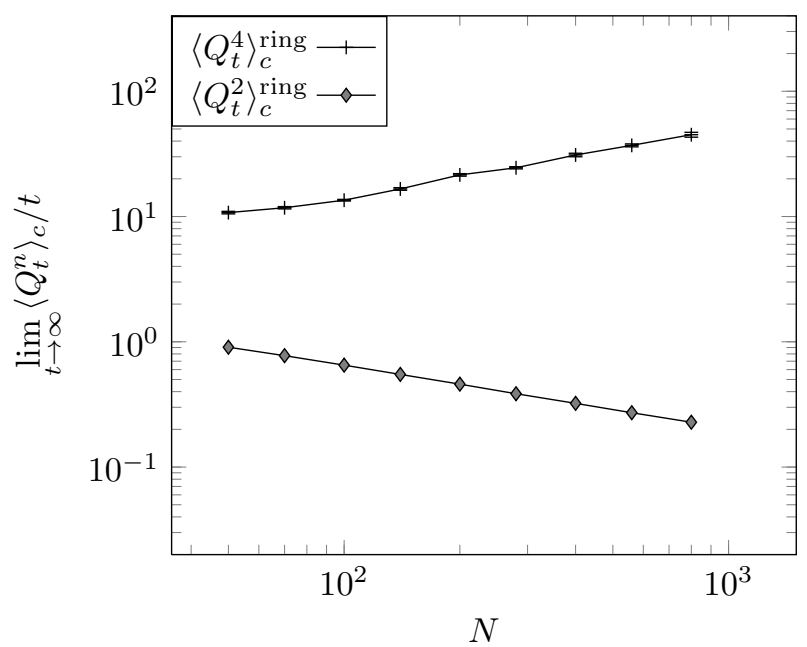

FIG. 3: Asymptotic values of $\left\langle Q_{t}^{n}\right\rangle_{c} / t$ on a ring of $50 \leq N \leq 800$ particles of alternating masses 1 and 2.62 , with total energy $N$ and total momentum 0 . While the second cumulant decreases as $N^{\alpha-1}$, with $\alpha \simeq 0.5$, the fourth cumulant increases with $N$. 
For the ring geometry, while the second cumulant decays like a power law, the fourth cumulant increases with the system size. Hence the picture is very different from the diffusive case 11: in addition to the anomalous Fourier's law, we observe an increase of the fourth cumulant instead of a decay.
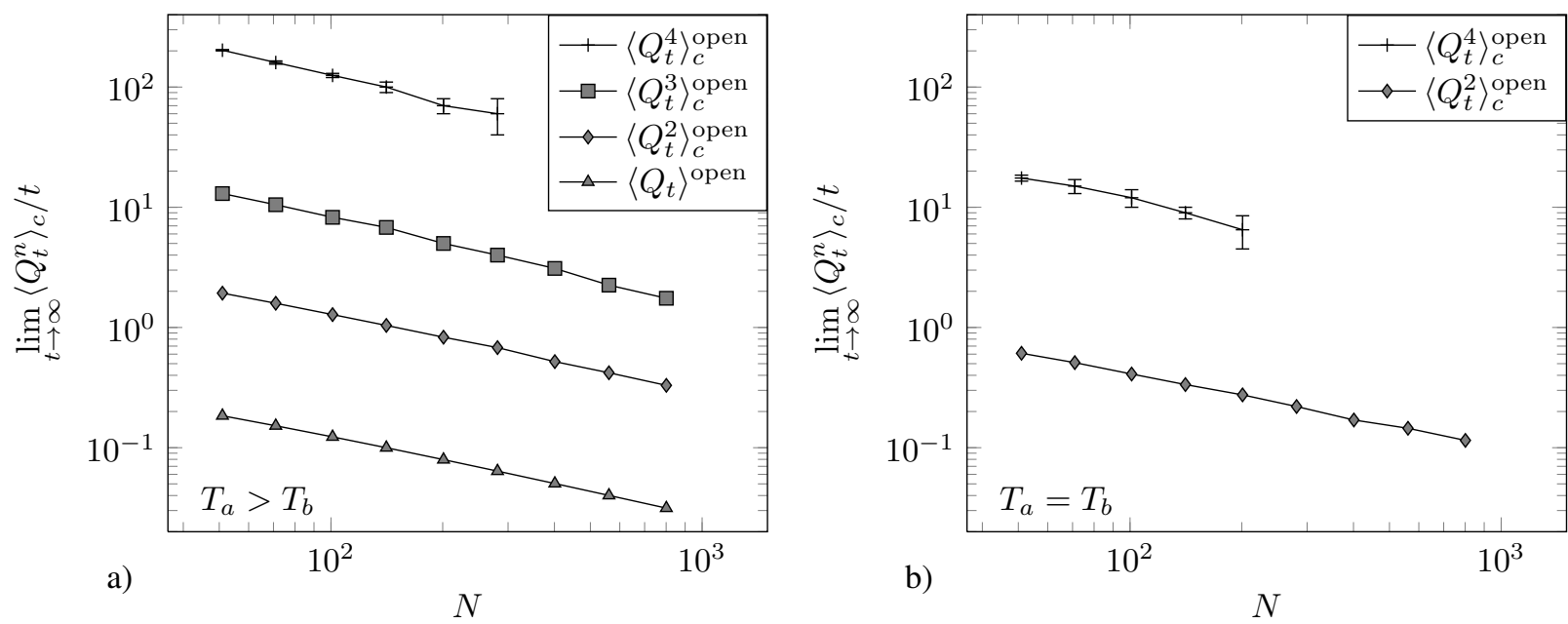

FIG. 4: Asymptotic values of $\left\langle Q_{t}^{n}\right\rangle_{c} / t$ on an open system with $51 \leq N \leq 801$ particles, with alternating masses 1 and 2.62 , between heat baths at temperatures $T_{a}=2$ and $T_{b}=1$ (fig. 4 a) and $T_{a}=T_{b}=1$ (fig. 4 b). All cumulants seem to decrease as $N^{\alpha-1}$, with $0.25 \leq \alpha \leq 0.4$.

For the open case, the situation looks more similar to the diffusive case : all the cumulants seem to decrease with comparable power laws of the system size, albeit with a different exponent than in the diffusive case (1).

\section{SHORT-TIME BEHAVIOR}
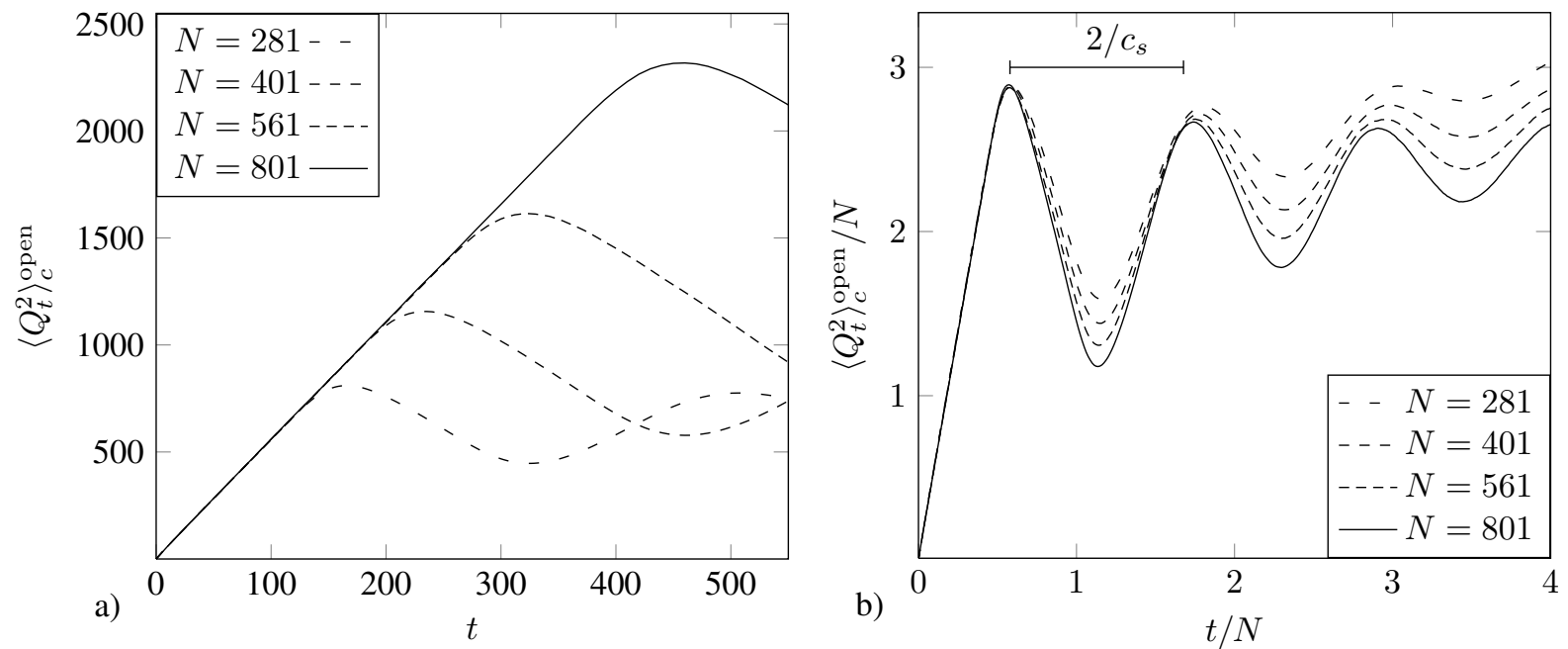

FIG. 5: Short-time behavior of $\left\langle Q_{t}^{2}\right\rangle_{c}$ on an open system of $281 \leq N \leq 801$ particles between heat baths at the same temperature $T_{a}=T_{b}=$ 1. In a time range increasing with system size, the second cumulant behaves as on an infinite system at equilibrium (fig. 5 a); it then exhibits damped oscilllations on time scales of order $N$ (fig. 5 b).

In our simulations (fig. 2]a), we measure the whole time dependence of the cumulants $\left\langle Q_{t}^{n}\right\rangle_{c}$. As fig. 5 shows, the short-time behavior of $\left\langle Q_{t}^{n}\right\rangle_{c}$ is independent of the system size $N$ over a time range which increases with $N$. This can be interpreted as the fact that, over this time range, the system behaves as if it was infinite : in turn, this allows us to study the behavior of the cumulants on an infinite system. Fig. 5]a) shows that, for an infinite system at equilibrium, $\left\langle Q_{t}^{2}\right\rangle_{c}$ grows linearly with time; other results (not shown here) indicate that $\left\langle Q_{t}^{4}\right\rangle_{c}$ is also linear in time for the same system. This is another major difference with infinite diffusive systems [41], for which all the cumulants of $Q_{t}$ grow asymptotically as $\sqrt{t}$. 
At intermediate times, all the $\left\langle Q_{t}^{n}\right\rangle_{c}$ exhibit periodic oscillations for $n \geq 2$, with a period proportional to system size (as can be seen for the second cumulant on fig. 5 b): for the second cumulant, they can be fitted by an exponentially damped sine function [42]. The periods can be understood from the adiabatic sound velocity $c_{s}$ in the hard particle gas, given by

$$
c_{s}=\sqrt{\frac{\gamma P}{\rho}} ;
$$

here, $\gamma=3$ (for an one-dimensional monoatomic gas), $P=N k T / L=2$ (since the average energy, $E / N=k T / 2$, is taken to be 1 ), and $\rho=\left(1+m_{2}\right) / 2$, so that $c_{s}=1.82$ for $m_{2}=2.62$. For the open system, the period is close to $2 N / c_{s}$ (shown on fig. [5 b), which is the time for a sound wave originating in $S$ to come back to $S$ in the same direction, having been reflected once on each boundary; for the ring, our data (not shown here) exhibits a period close to $N / c_{s}$, the time for a sound wave starting from $S$ to go around the ring.

\section{CONCLUSION}

In this letter, we have shown that, for a hard particle gas at equilibrium and out of equilibrium, the cumulants of the flux of energy scale as power laws of the system size. These power laws differ from the ones expected for diffusive systems. Hence, our data shows that the anomalous Fourier's law of momentum-conserving systems is also characteristic of higher cumulants of the current. The difference between momentum-conserving and diffusive systems can also be seen for an infinite system at equilibrium, in which $\left\langle Q_{t}^{2}\right\rangle$ grows as $t$ instead of $\sqrt{t}$.

More numerical work is certainly needed to accurately determine the exponents characteristic of the cumulants of the energy flux, in particular to check whether, in the open case, all the cumulants decay with the same power law; one could try to compare the exponents seen for the ring and for the open geometry.

We have focused here on the fluctuations of the flux of energy, which is one of the conserved quantities of this hard particle gas. As momentum is also conserved, it would be interesting to study the size dependence of the fluctuations of the momentum flux in a similar way.

It would also be interesting to investigate whether similar power laws of the cumulants can be observed in other momentumconserving systems, such as anharmonic chains like the Fermi-Pasta-Ulam models (which are known to exhibit anomalous Fourier's law). The study of the cumulants could be a good test for existing theoretical approaches [43, 44], such as the modecoupling theory [45-48]. or the Boltzmann-Langevin equation [49, 50]. In particular, one may wonder whether, for momentumconserving systems, there exists an analog of the universal ratios of the cumulants which are expected for diffusive systems on a ring [10].

[1] D. J. Evans, E. G. D. Cohen, and G. P. Morriss, "Probability of second law violations in shearing steady states," Phys. Rev. Lett., vol. 71, p. 3616, Nov 1993.

[2] G. Gallavotti and E. G. D. Cohen, "Dynamical ensembles in nonequilibrium statistical mechanics," Phys. Rev. Lett., vol. 74, pp. 26942697, Apr 1995.

[3] G. Gallavotti and E. Cohen, "Dynamical ensembles in stationary states," J. Stat. Phys., vol. 80, pp. 931-970, Sep 1995.

[4] D. J. Evans and D. J. Searles, "The fluctuation theorem," Adv. Phys., vol. 51, no. 7, pp. 1529-1585, 2002.

[5] C. Maes, “The fluctuation theorem as a gibbs property,” J. Stat. Phys., vol. 95, pp. 367-392, Apr 1999.

[6] R. J. Harris and G. M. Schutz, "Fluctuation theorems for stochastic dynamics," J. Stat. Mech: Theory Exp., vol. 2007, no. 07, p. P07020.

[7] T. Bodineau and B. Derrida, "Current fluctuations in nonequilibrium diffusive systems: An additivity principle," Phys. Rev. Lett., vol. 92, p. 180601, May 2004.

[8] L. Bertini, A. De Sole, D. Gabrielli, G. Jona-Lasinio, and C. Landim, "Current fluctuations in stochastic lattice gases," Phys. Rev. Lett., vol. 94, p. 030601, Jan 2005.

[9] L. Bertini, A. Sole, D. Gabrielli, G. Jona-Lasinio, and C. Landim, "Non equilibrium current fluctuations in stochastic lattice gases," J. Stat. Phys., vol. 123, pp. 237-276, Apr 2006.

[10] C. Appert-Rolland, B. Derrida, V. Lecomte, and F. van Wijland, "Universal cumulants of the current in diffusive systems on a ring," Phys. Rev. E, vol. 78, p. 021122, Aug 2008.

[11] P. I. Hurtado and P. L. Garrido, "Current fluctuations and statistics during a large deviation event in an exactly solvable transport model," J. Stat. Mech: Theory Exp., vol. 2009, no. 02, p. P02032.

[12] A. Imparato, V. Lecomte, and F. van Wijland, "Equilibriumlike fluctuations in some boundary-driven open diffusive systems," Phys. Rev. E, vol. 80, p. 011131, Jul 2009.

[13] R. J. Harris, A. Rakos, and G. M. Schutz, "Current fluctuations in the zero-range process with open boundaries," J. Stat. Mech: Theory Exp., vol. 2005, no. 08, p. P08003. 
[14] B. Derrida, "Non-equilibrium steady states: fluctuations and large deviations of the density and of the current," J. Stat. Mech: Theory Exp., vol. 2007, no. 07, p. P07023.

[15] H. Lee, L. S. Levitov, and A. Y. Yakovets, "Universal statistics of transport in disordered conductors," Phys. Rev. B, vol. 51, pp. 40794083, Feb 1995.

[16] A. N. Jordan, E. V. Sukhorukov, and S. Pilgram, "Fluctuation statistics in networks: A stochastic path integral approach," Journal of Mathematical Physics, vol. 45, no. 11, pp. 4386-4417, 2004.

[17] C. Kipnis, C. Marchioro, and E. Presutti, "Heat flow in an exactly solvable model," J. Stat. Phys., vol. 27 , pp. 65-74, Jan 1982.

[18] B. Derrida, B. Douçot, and P.-E. Roche, "Current fluctuations in the one-dimensional symmetric exclusion process with open boundaries," J. Stat. Phys., vol. 115, pp. 717-748, May 2004.

[19] S. Prolhac and K. Mallick, "Cumulants of the current in a weakly asymmetric exclusion process," J. Phys. A: Math. Theor., vol. 42, no. 17, p. 175001, 2009.

[20] F. Bonetto, J. L. Lebowitz, and L. Rey-Bellet, "Fourier's law: a challenge to theorists," in Mathematical physics 2000, pp. 128-150, London: Imp. Coll. Press, 2000.

[21] C. Kipnis, S. Olla, and S. R. S. Varadhan, "Hydrodynamics and large deviation for simple exclusion processes," Communications on Pure and Applied Mathematics, vol. 42, no. 2, pp. 115-137, 1989.

[22] H. Spohn, Large Scale Dynamics of Interacting Particles. Texts and Monographs in Physics, Springer, November 1991.

[23] L. Bertini, A. De Sole, D. Gabrielli, G. Jona-Lasinio, and C. Landim, "Fluctuations in stationary nonequilibrium states of irreversible processes," Phys. Rev. Lett., vol. 87, p. 040601, Jul 2001.

[24] L. Bertini, A. De Sole, D. Gabrielli, G. Jona-Lasinio, and C. Landim, "Macroscopic fluctuation theory for stationary non-equilibrium states," J. Stat. Phys., vol. 107, pp. 635-675, May 2002.

[25] L. Bertini, A. De Sole, D. Gabrielli, G. Jona-Lasinio, and C. Landim, "Large deviation approach to non equilibrium processes in stochastic lattice gases," Bulletin of the Brazilian Mathematical Society, vol. 37, pp. 611-643, Dec 2006.

[26] S. Lepri, R. Livi, and A. Politi, "Heat conduction in chains of nonlinear oscillators," Phys. Rev. Lett., vol. 78, pp. 1896-1899, Mar 1997.

[27] S. Lepri, R. Livi, and A. Politi, "Thermal conduction in classical low-dimensional lattices," Phys. Rep., vol. 377, pp. 1-80(80), April 2003.

[28] A. Dhar, "Heat transport in low-dimensional systems," Adv. Phys., vol. 57, no. 5, pp. 457-537, 2008.

[29] T. c. v. Prosen and D. K. Campbell, "Momentum conservation implies anomalous energy transport in 1d classical lattices," Phys. Rev. Lett., vol. 84, pp. 2857-2860, Mar 2000.

[30] B. Li and J. Wang, "Anomalous heat conduction and anomalous diffusion in one-dimensional systems," Phys. Rev. Lett., vol. 91, p. 044301, Jul 2003.

[31] G. Basile, C. Bernardin, and S. Olla, "Momentum conserving model with anomalous thermal conductivity in low dimensional systems," Phys. Rev. Lett., vol. 96, p. 204303, May 2006.

[32] T. Mai, A. Dhar, and O. Narayan, "Equilibration and universal heat conduction in fermi-pasta-ulam chains," Phys. Rev. Lett., vol. 98, p. 184301, May 2007.

[33] G. Casati and T. c. v. Prosen, "Anomalous heat conduction in a one-dimensional ideal gas," Phys. Rev. E, vol. 67, p. 015203 , Jan 2003.

[34] S. Lepri, R. Livi, and A. Politi, "Universality of anomalous one-dimensional heat conductivity," Phys. Rev. E, vol. 68, p. 067102, Dec 2003.

[35] P. Grassberger, W. Nadler, and L. Yang, "Heat conduction and entropy production in a one-dimensional hard-particle gas," Phys. Rev. Lett., vol. 89, p. 180601, Oct 2002.

[36] A. Dhar, "Heat conduction in a one-dimensional gas of elastically colliding particles of unequal masses," Phys. Rev. Lett., vol. 86, pp. 3554-3557, Apr 2001.

[37] A. Dhar and O. Narayan, "Dhar et al. reply:," Phys. Rev. Lett., vol. 100, p. 199402, May 2008.

[38] O. Narayan and S. Ramaswamy, "Anomalous heat conduction in one-dimensional momentum-conserving systems," Phys. Rev. Lett., vol. 89, p. 200601, Oct 2002.

[39] G. Basile, L. Delfini, S. Lepri, R. Livi, S. Olla, and A. Politi, "Anomalous transport and relaxation in classical one-dimensional models," Eur. Phys. J. Special Topics, vol. 151, pp. 85-93, dec 2007.

[40] J. M. Deutsch and O. Narayan, "One-dimensional heat conductivity exponent from a random collision model," Phys. Rev. E, vol. 68, p. 010201, Jul 2003.

[41] B. Derrida and A. Gerschenfeld, "Current fluctuations in one dimensional diffusive systems with a step initial density profile," J. Stat. Phys., vol. 137, pp. 978-1000, Dec 2009.

[42] M. Bishop, "Collective modes of one-dimensional Lennard-Jones systems," J. Stat. Phys., vol. 29, pp. 623-629, Nov 1982.

[43] T. Mai and O. Narayan, "Universality of one-dimensional heat conductivity," Phys. Rev. E, vol. 73, p. 061202 , Jun 2006.

[44] J. Lukkarinen and H. Spohn, "Anomalous energy transport in the FPU- $\beta$ chain," Comm. Pure Appl. Math., vol. 61, no. 12, pp. 1753-1786, 2008.

[45] Y. Pomeau and P. Résibois, “Time dependent correlation functions and mode-mode coupling theories,” Phys. Rep., vol. 19, no. 2, pp. 63 $-139,1975$.

[46] S. Lepri, "Relaxation of classical many-body hamiltonians in one dimension," Phys. Rev. E, vol. 58, pp. 7165-7171, Dec 1998.

[47] L. Delfini, S. Lepri, R. Livi, and A. Politi, "Anomalous kinetics and transport from 1d self-consistent mode-coupling theory," J. Stat. Mech: Theory Exp., vol. 2007, no. 02, p. P02007.

[48] J.-S. Wang and B. Li, "Mode-coupling theory and molecular dynamics simulation for heat conduction in a chain with transverse motions," Phys. Rev. E, vol. 70, p. 021204, Aug 2004.

[49] M. Bixon and R. Zwanzig, "Boltzmann-Langevin equation and hydrodynamic fluctuations," Phys. Rev., vol. 187, pp. $267-272$, Nov 1969.

[50] A. Pereverzev, "Fermi-Pasta-Ulam $\beta$ lattice: Peierls equation and anomalous heat conductivity," Phys. Rev. E, vol. 68, p. 056124, Nov 2003. 